Supporting Information

\title{
Nickel-Catalyzed Synthesis of Benzocoumarins: Application to the Total Synthesis of Arnottin I
}

\author{
Sachin Madan and Chien-Hong Cheng* \\ Department of Chemistry, National Tsing Hua University, Hsinchu 30013, Taiwan \\ chcheng@mx.nthu.edu.tw
}

Experimental Procedures and Spectral Data S2

${ }^{1} \mathrm{H}$ NMR spectra of 3

S3

${ }^{1} \mathrm{H}$ NMR spectra of 5

S4

${ }^{1} \mathrm{H}$ NMR spectra of $\mathbf{6}$

S5

${ }^{1} \mathrm{H}$ NMR spectra of 7

S6

${ }^{1} \mathrm{H}$ NMR spectra of $\mathbf{9}$

S7

${ }^{1} \mathrm{H}$ NMR spectra of $\mathbf{1 0}$

S8

${ }^{1} \mathrm{H}$ NMR spectra of $\mathbf{1 1}$

S9

${ }^{1} \mathrm{H}$ NMR spectra of $\mathbf{1 2}$

S10

${ }^{1} \mathrm{H}$ NMR spectra of $\mathbf{1 3}$

S11

${ }^{1} \mathrm{H}$ NMR spectra of $\mathbf{1 4}$

S12

${ }^{1} \mathrm{H}$ NMR spectra of $\mathbf{1 5}$

S13

${ }^{1} \mathrm{H}$ NMR spectra of $\mathbf{1 6}$

S14 
1,2-Bis(allyloxy)-4,5-dibromobenzene. 4,5-Dibromocatechol (1.34 g, $5.0 \mathrm{mmol}$ ) was dissolved in dry THF (15 mL) and to this, NaH (440 mg, $11.0 \mathrm{mmol}$, 60\% suspension in oil) was added and mixture was stirred for $15 \mathrm{~min}$. Allyl iodide (2.5 g, $1.4 \mathrm{~mL}, 15 \mathrm{mmol})$ was added to the above mixture dropwise and the reaction mixture was stirred at room temperature for $15 \mathrm{~h}$. It was quenched with water, brine and extracted with diethyl ether $(4 \times 10 \mathrm{~mL})$. The combined organic layers were washed with brine and dried over $\mathrm{MgSO}_{4}$. The crude product was then concentrated in vacuo followed by silica gel column using (ethyl acetate:hexanes, 1:20) to give 1.36 g of 1,2-bis(allyloxy)-4,5-dibromobenzene (78\%): ${ }^{1} \mathrm{H}$ NMR (600 MHz, $\left.\mathrm{CDCl}_{3}\right) \delta 4.53$ (m, 4H), $5.27(\mathrm{~m}, 2 \mathrm{H}), 5.38(\mathrm{~m}, 2 \mathrm{H}), 6.01(\mathrm{~m}, 2 \mathrm{H}), 7.06(\mathrm{~s}, 2 \mathrm{H}) ;{ }^{13} \mathrm{C}$ NMR (150 MHz, $\left.\mathrm{CDCl}_{3}\right)$ $\delta$ 70.14, 115.01, 118.22, 118.40, 132.39, 148.29.

1,2-Bis(tert-butyldimethylsilyloxy)-4,5-dibromobenzene. 4,5-Dibromocatechol (1.34 g, 5 mmol) was dissolved in DMF (15 mL) and to this, tert-butylchlorodimethylsilane (2.25 g, 15 mmol) and imidazole (1.7 g, $25 \mathrm{mmol})$ were added and the mixture was stirred at room temperature for overnight. It was quenched with water and extracted with methylene chloride (5 x $10 \mathrm{~mL}$ ). The combined organic layers were washed with brine and dried over $\mathrm{MgSO}_{4}$. The crude product was then concentrated in vacuo followed by short silica gel column using hexanes to give $2.1 \mathrm{~g}$ of 1 (85\%): ${ }^{1} \mathrm{H}$ NMR (600 MHz, $\left.\mathrm{CDCl}_{3}\right) \delta 0.18$ (s, 12H), 0.95 (s, 18H), 7.03 (s, $2 \mathrm{H}) ;{ }^{13} \mathrm{C}$ NMR $\left(150 \mathrm{MHz}, \mathrm{CDCl}_{3}\right) \delta-4.01\left(\mathrm{CH}_{3}-\mathrm{Si}\right), 17.94,26.77,117.88,130.29,148.03$. 


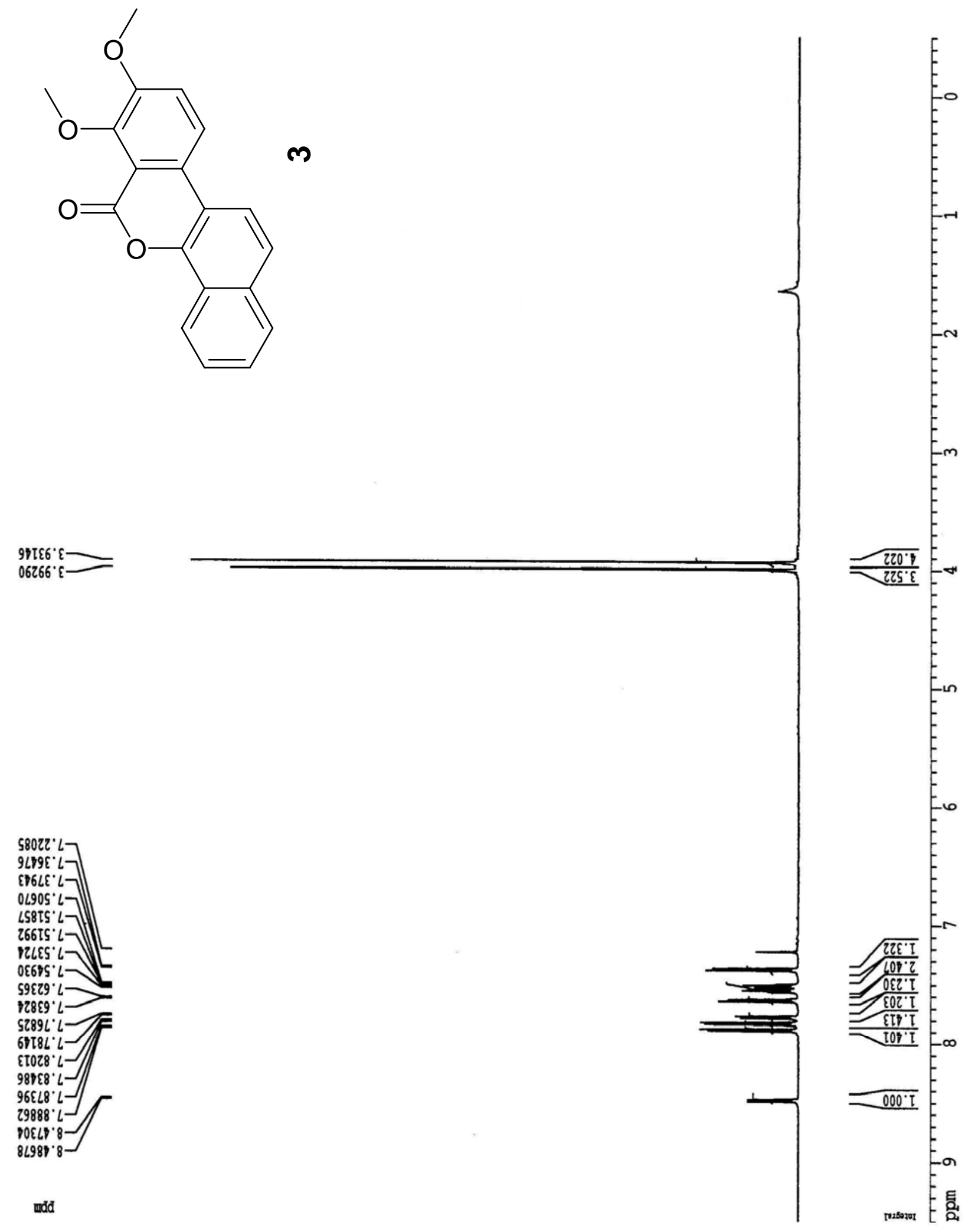




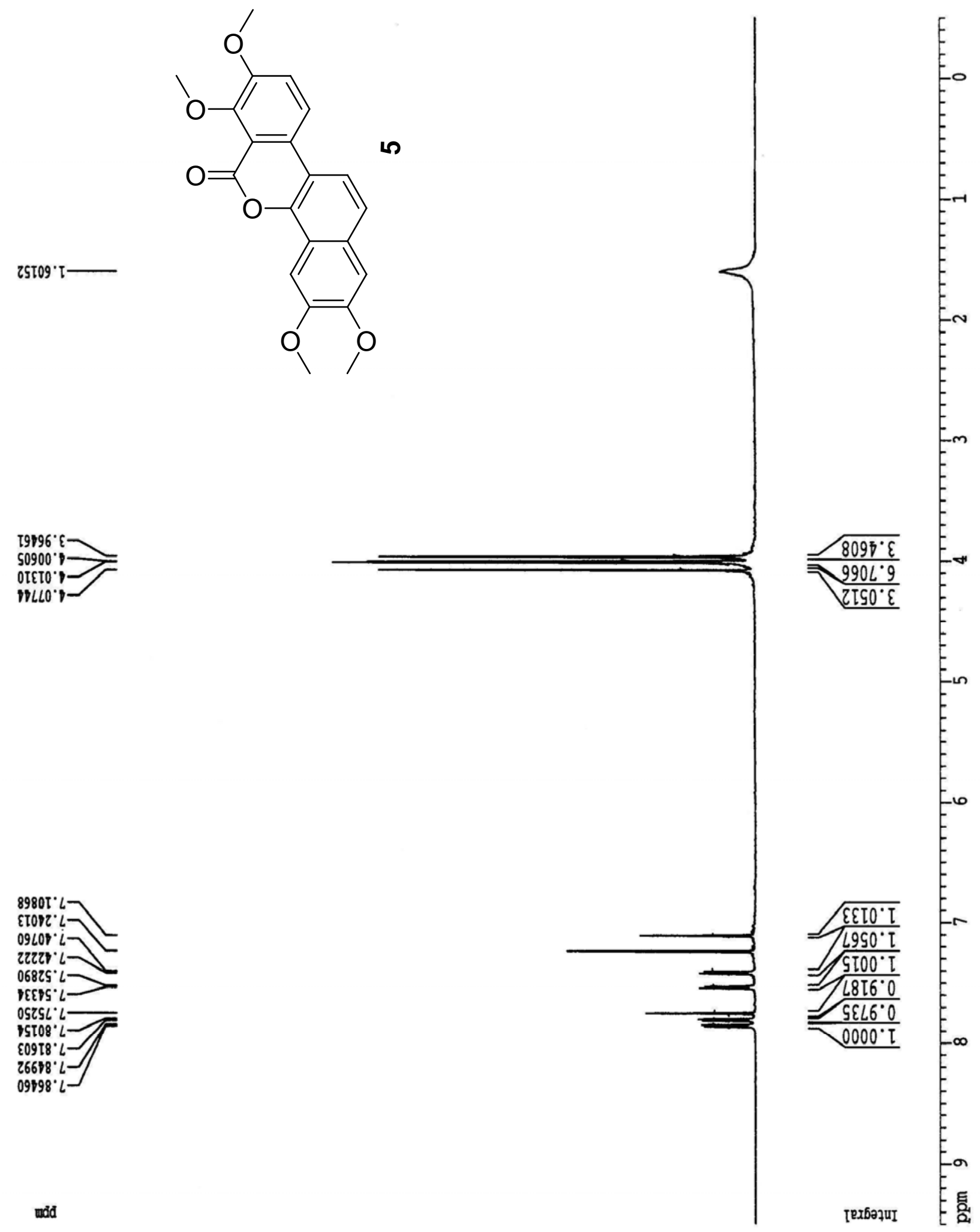


825. I-

$229 \cdot 5-$

$0<8^{\circ} \mathrm{s}$

I26.5 -

5โ8.9-

$820.2-$

$002 \times 2$
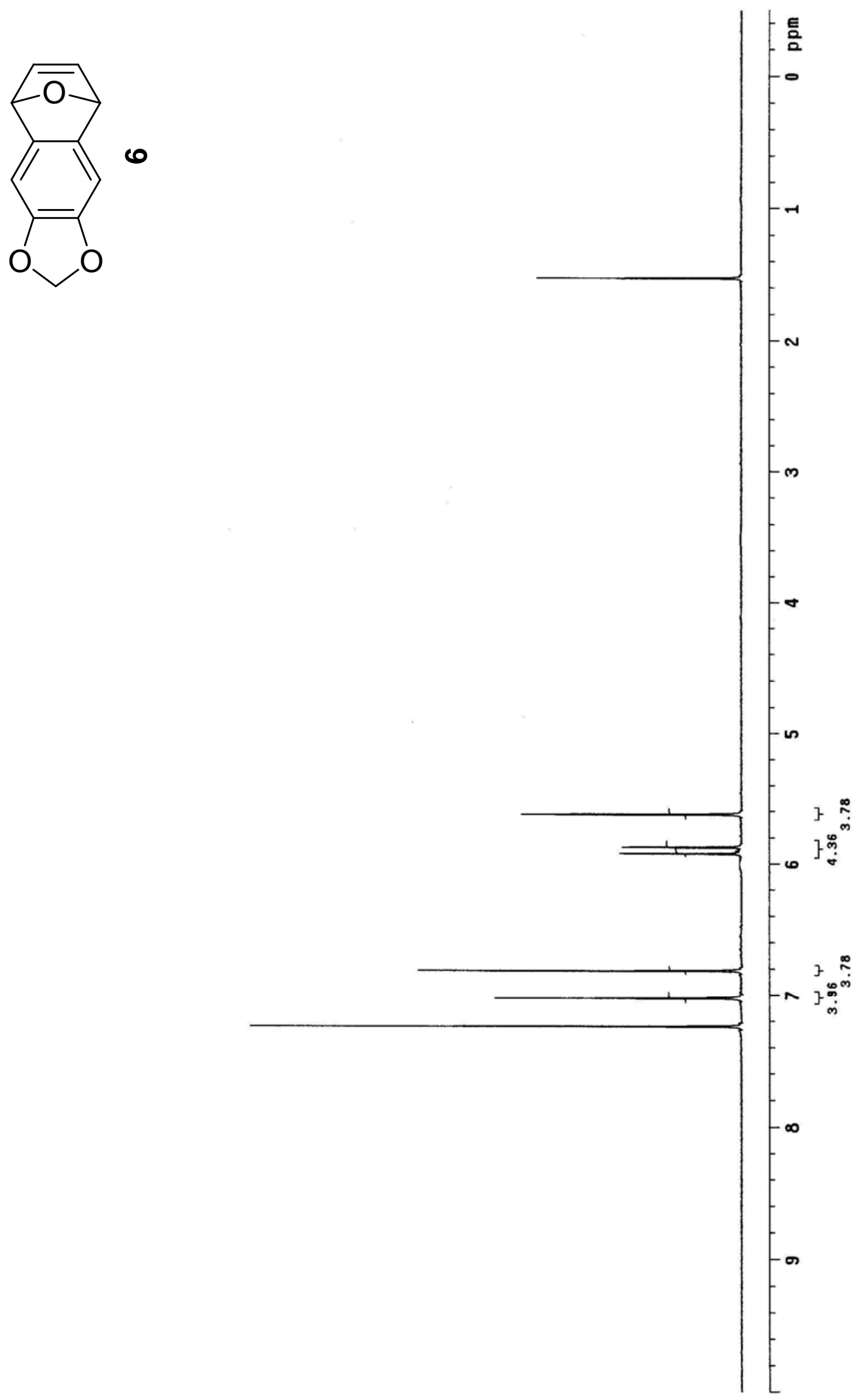


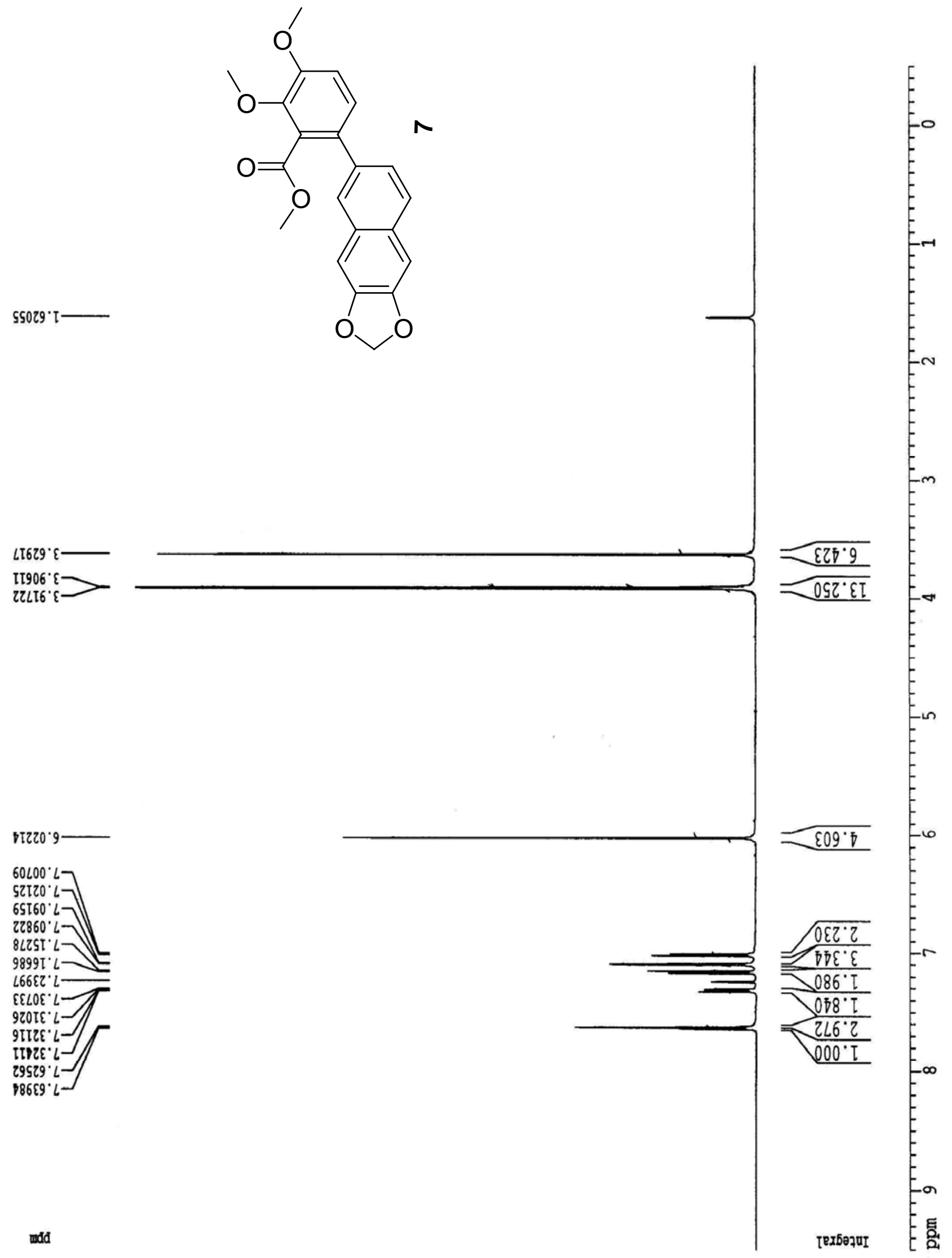




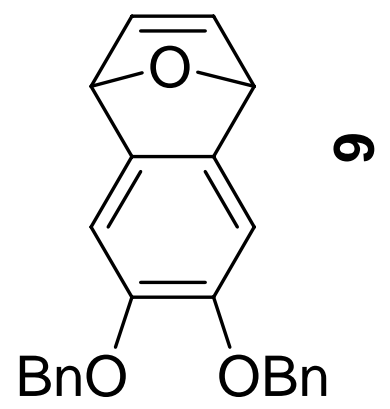

I60.s-

6I9.s-

686.97

062.27

or $6^{\circ} 2$

$088^{\circ} \mathrm{L}$

$098^{\circ} \mathrm{L}$

$298^{\circ} \mathrm{L}$

$020^{\circ}<2$

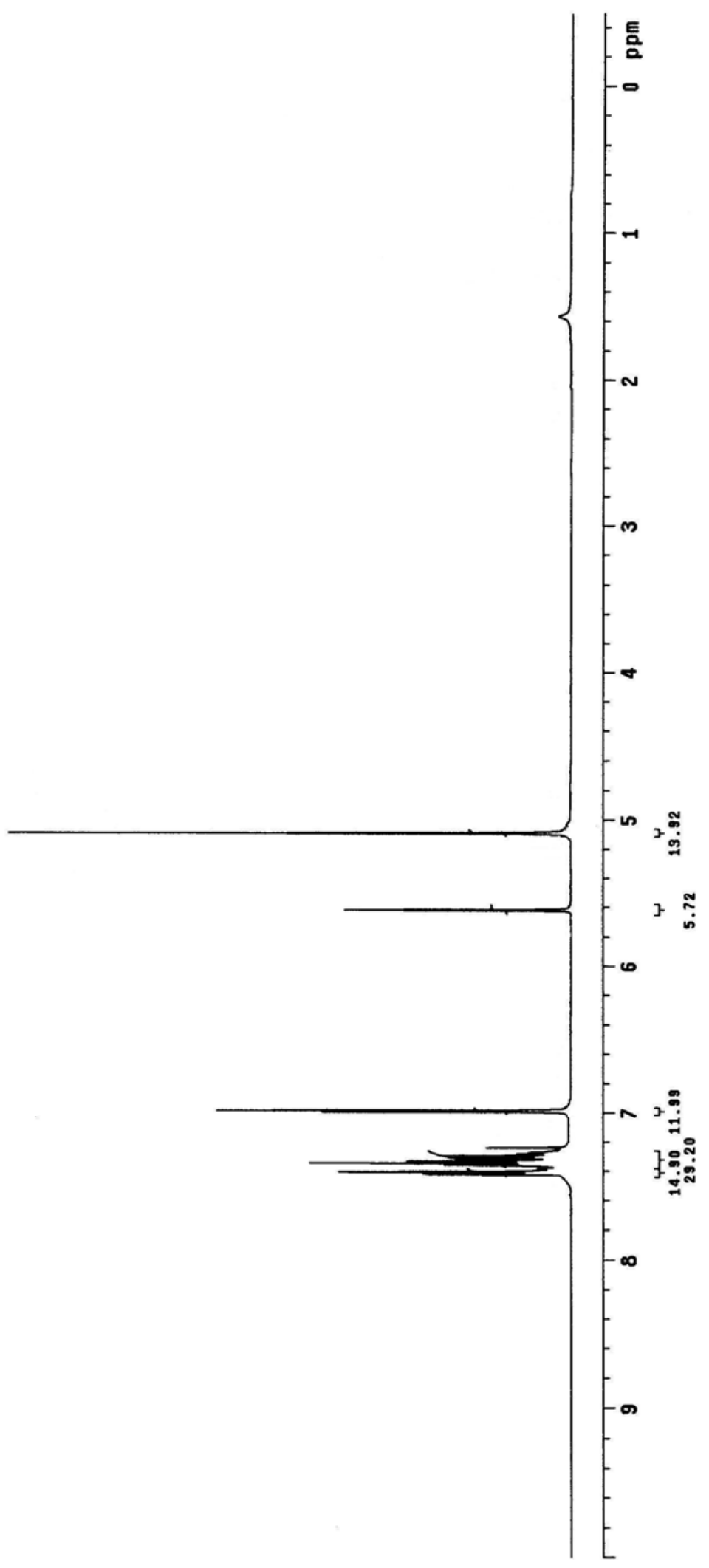


622.

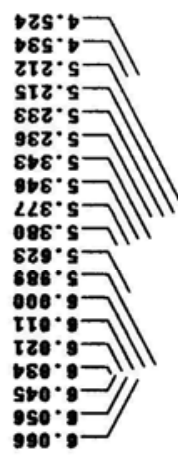

286.9工

002.2

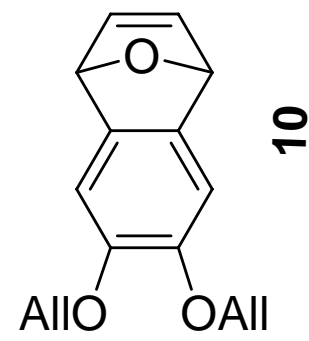

어

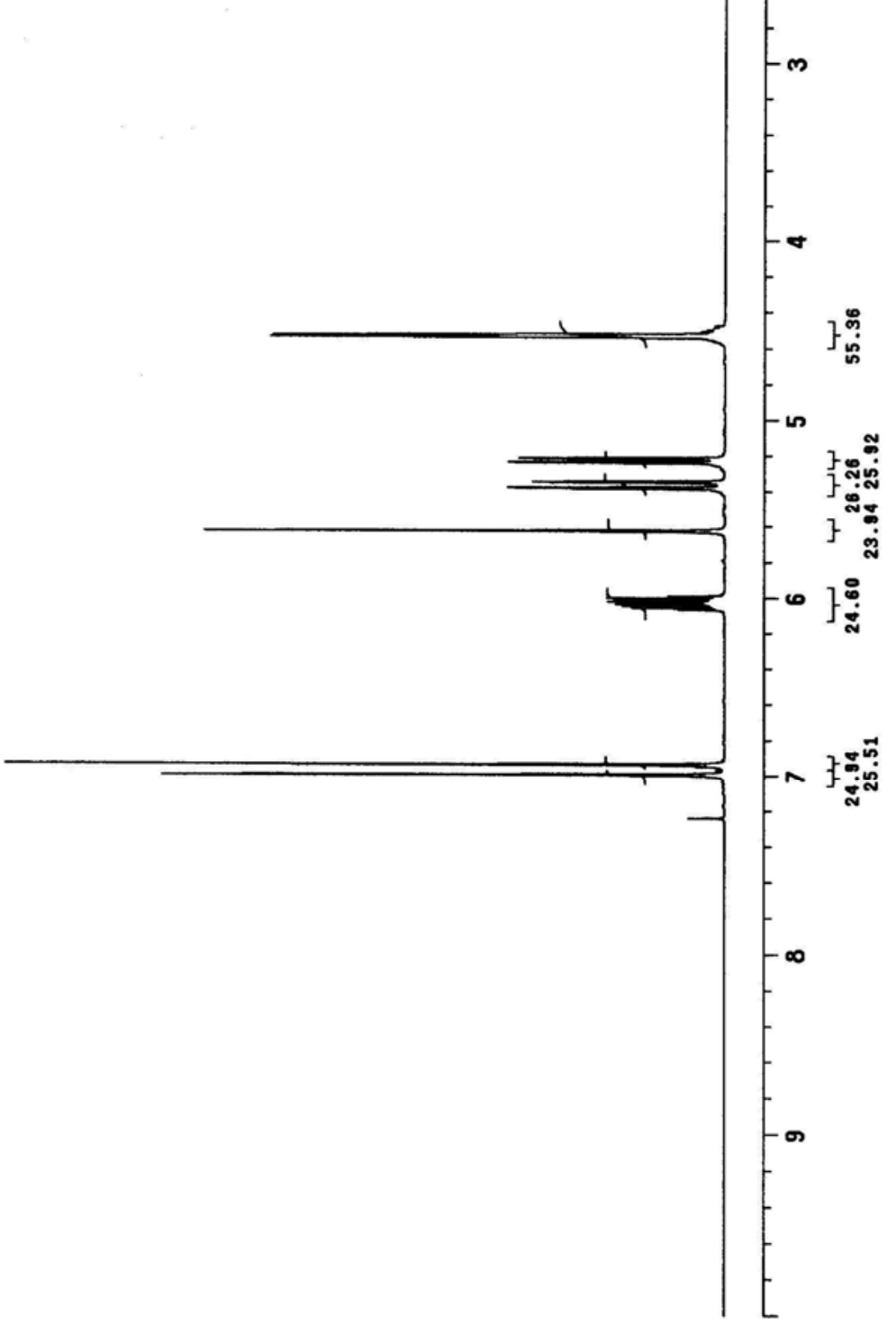




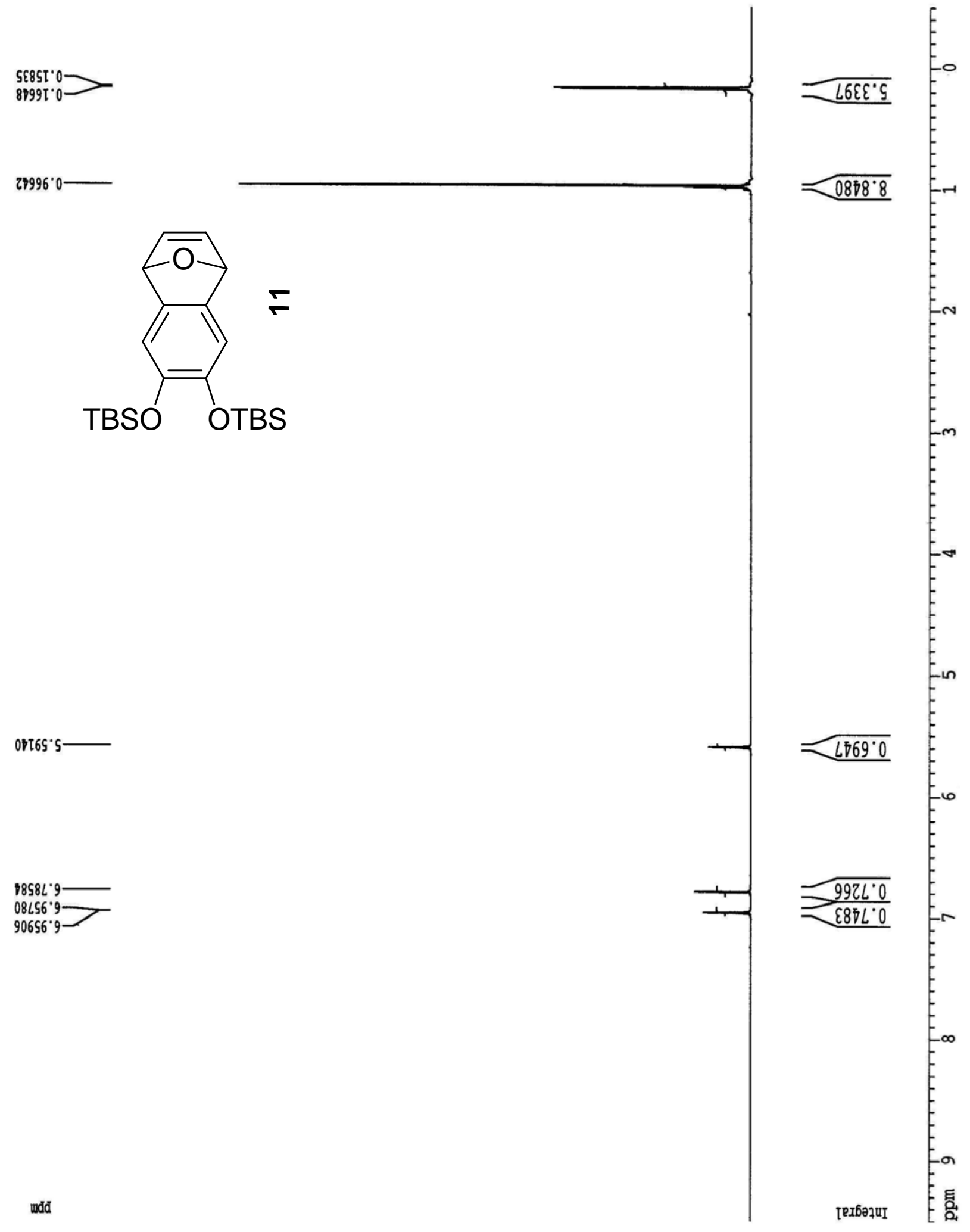




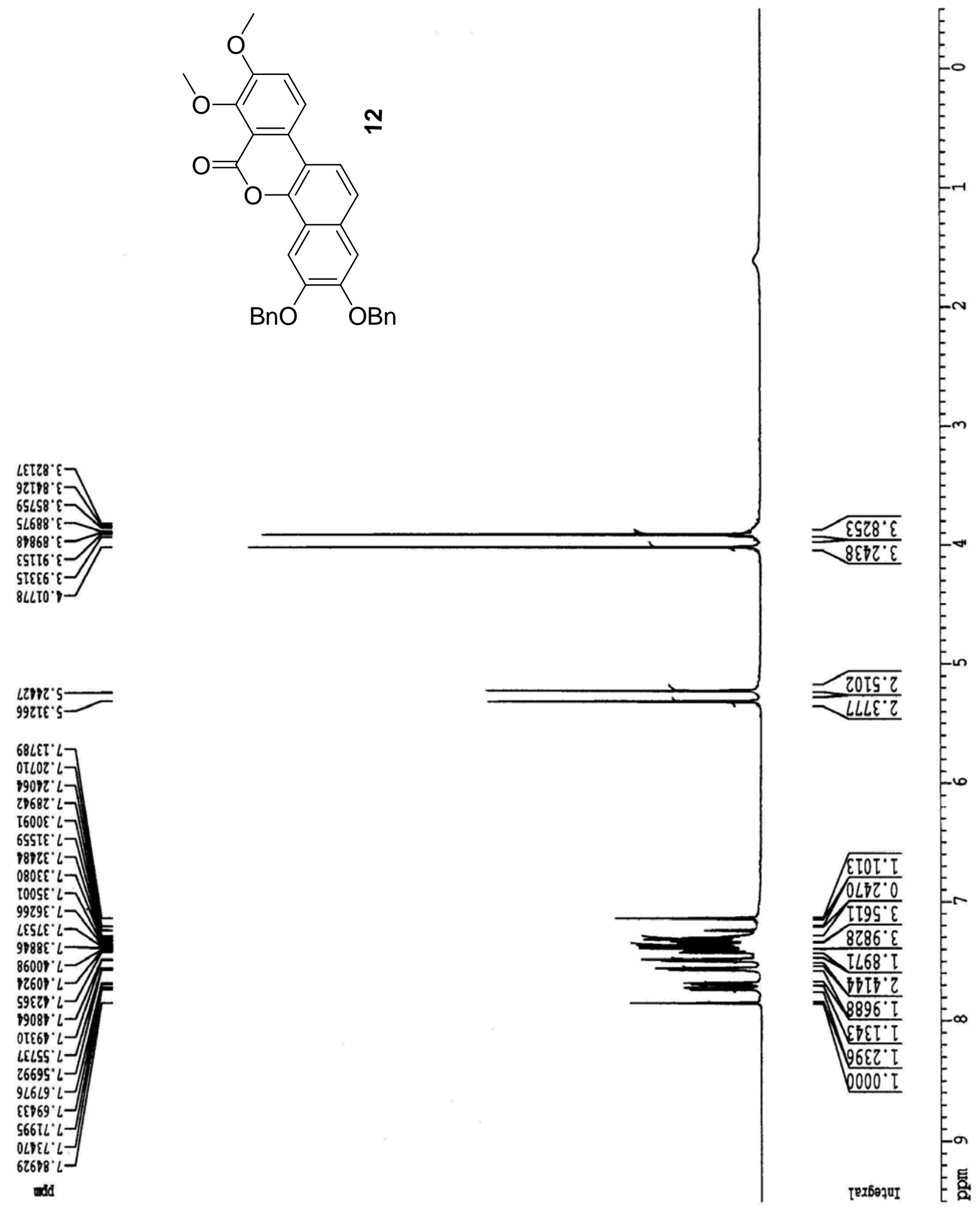


$95885^{\circ} \mathrm{-}$<smiles>COc1cc2ccc3c4ccc(O)c(OC)c4c(=O)oc3c2cc1O</smiles>

OIIS6 ' $\varepsilon-$

72666 ' $\varepsilon$

$26500^{\circ} \mathrm{7}$

$9980 L^{\prime}>$

EELIL'

เท8L'

LIร6L'

288te's 5 E

groze's-

દ8ระโ' '

ZIISE' $S$

हกเระ. 5

STIL'S-

LLLA's] E

L6BOS'S

โS905's-

zoszs's

26925's

$18555^{\circ} 5$

$85555^{\circ} 5$

$6 \mathrm{LLTI} \cdot 9$

7092T' 9

$825 \pi \cdot 9$

06ESt' 9

8LZ9I'9

OrILI' 9

96 เ8 '9

00T6I' 9

2866 '9

$0020 T^{\circ} \mathrm{L}$

โ86โ2 $L$

828LE' $L$

$6626 \AA^{\circ} \mathrm{L}$

SLELP. $L$

I $288^{\circ} \mathrm{L}-$

$62911 \circ$

OSSSL $L$

IZOLL $L$

$85608^{\circ} \mathrm{L}-$

9โจ $28^{\circ}\llcorner$

wdd

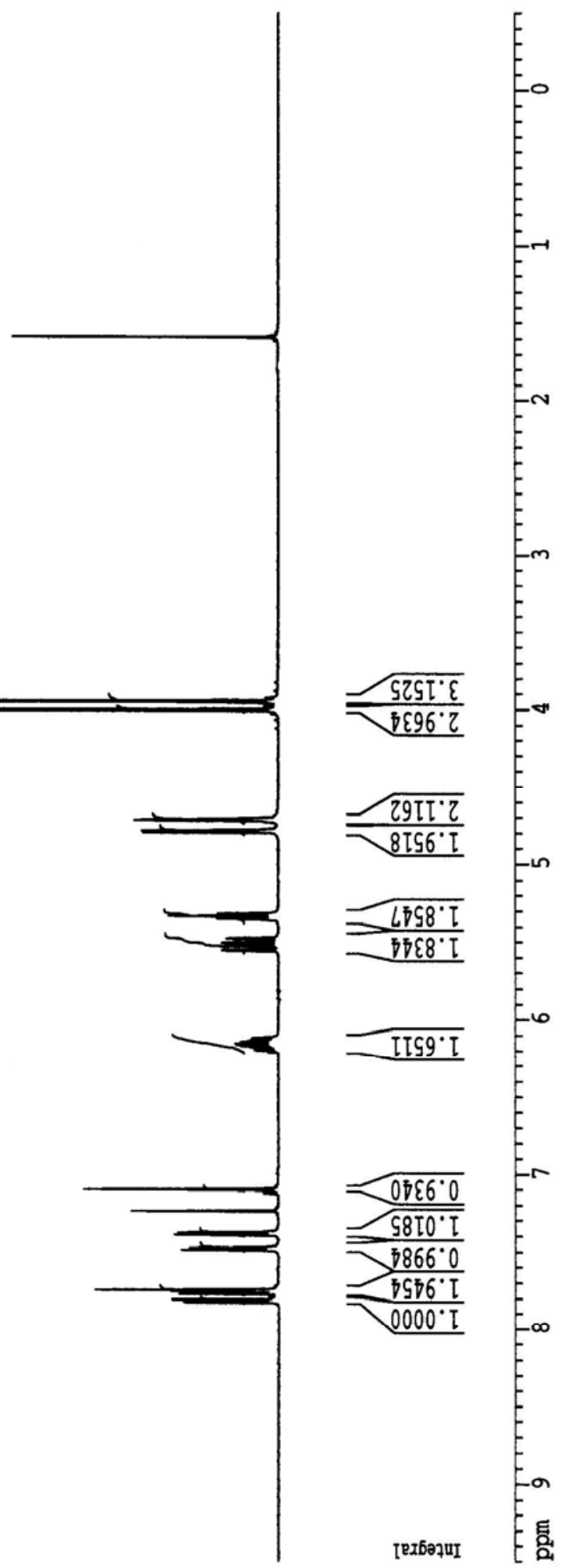




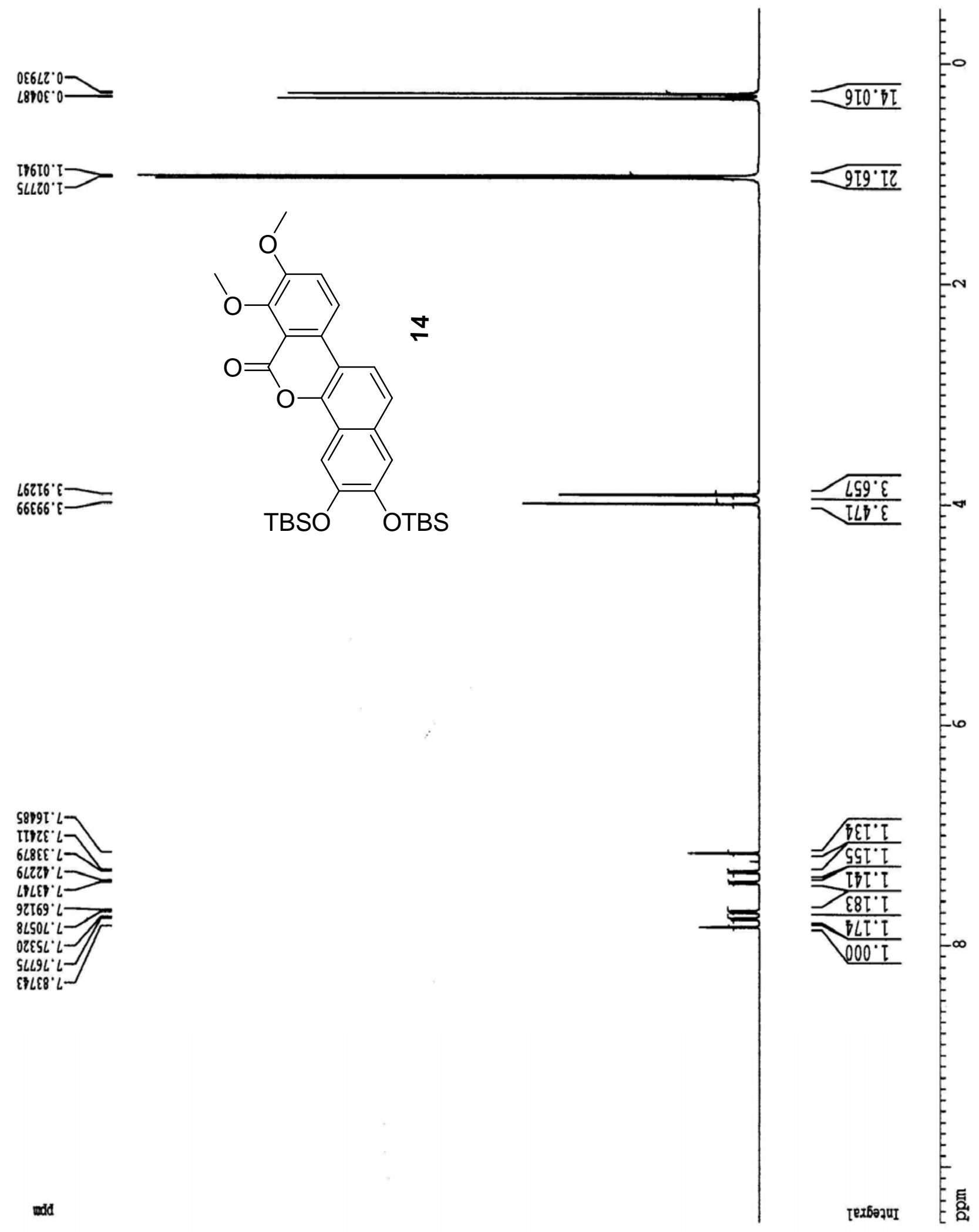




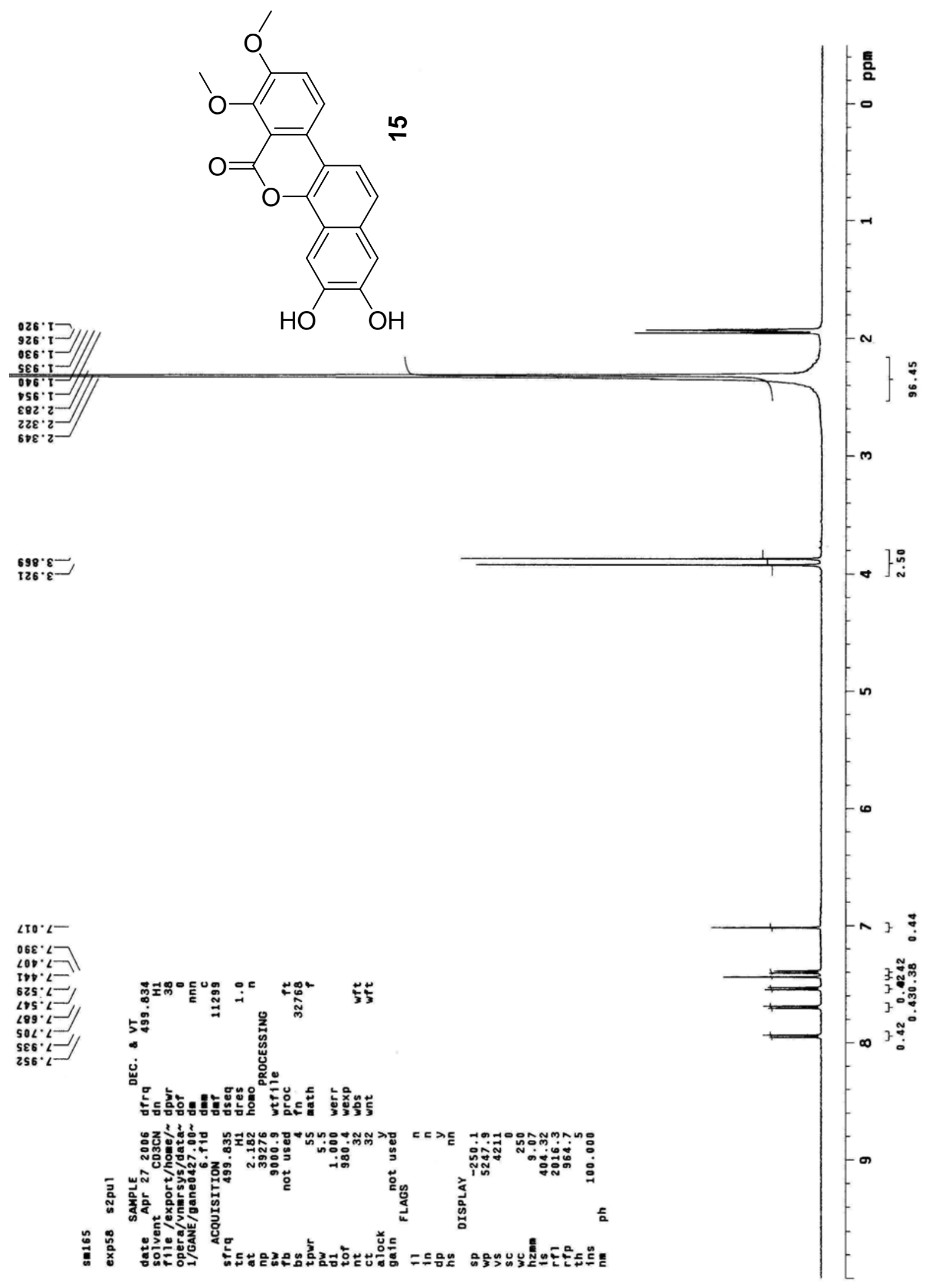




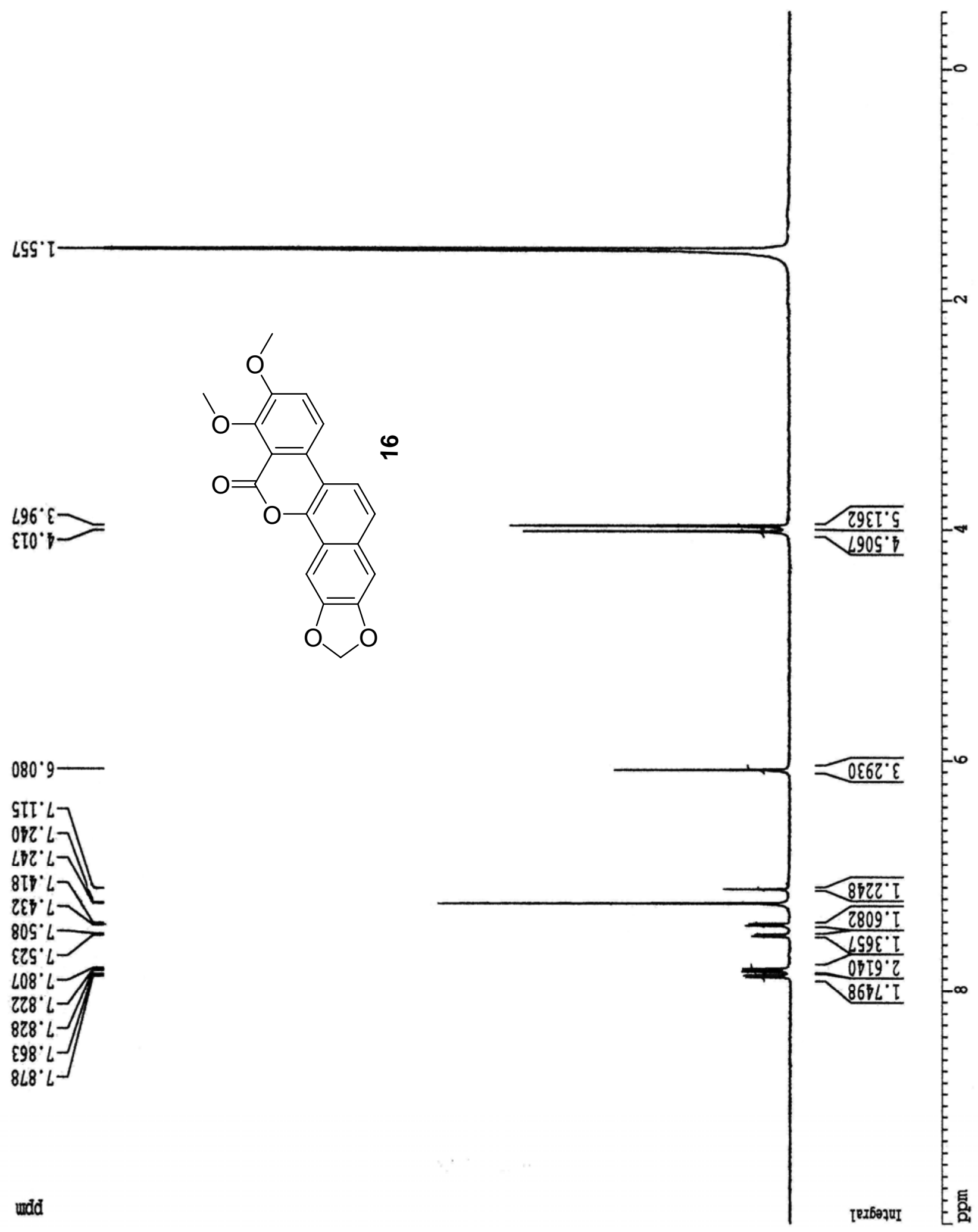

